\title{
FARMÁCIA DOMICILIAR: UMA CAIXA DE SURPRESAS.
}

\section{HOME DRUGSTORE: THE FULL BOX SURPRISE}

SANTIN, Patrícia Oliveira Rocha'; VIRTUOSO, Suzane*2; DE OLIVEIRA, Simone Maria Menegatti

1 - Farmacêutica - Parte da Monografia de Especialização em Farmacologia Aplicada (UNIOESTE - PR)

2 - Professora de Atenção Farmacêutica - Curso de Farmácia - UNIOESTE - Orientadora

3 - Professora de Estágio em Farmácia de Manipulação - Curso de Farmácia - UNIOESTE - Co-orientadora

*email: suzane@unioeste.br

RECBIDO: JUN/07 ACEITE: SET/07

\begin{abstract}
RESUMO
O intuito do estudo é fornecer à comunidade orientação e informação sobre cada medicamento que possuem em casa e indiretamente demonstrar aos clientes de farmácia comercial o quão importante é utilizar-se dos conhecimentos do farmacêutico que realiza a atenção farmacêutica, um serviço ainda pouco conhecido. Para a realização da pesquisa, realizou-se o convite aos clientes em uma farmácia comercial do município de Cascavel-PR, esclarecendo-os sobre o objetivo do mesmo, para os quinze primeiros aceites foi explicado o termo de consentimento livre e esclarecido e havendo concordância, marcou-se um horário para o atendimento que foi realizado no escritório da farmácia. A análise baseouse na "fase da sacola de medicamentos" do método Dadér de acompanhamento farmacoterapêutico. No modo geral, a comunidade apresentou certa desenvoltura no conhecimento da sua caixinha de medicamentos domiciliares e interesse pelo serviço diferenciado proposto.
\end{abstract}

Palavras-chave: medicamento domiciliar; farmacêutico; atenção farmacêutica.

\begin{abstract}
The intention of the study is to supply to the community orientation and information about each medicine that have in their house and indirectly to demonstrate to the commercial pharmacy customers the importance of the pharmacist knowledge who carries through pharmaceutical attention, a service that is not still very known. For the accomplishment of the research the invitation to the customers in a commercial pharmacy of Cascavel-PR city was made clarifying them on this objective, for the fifteen first acceptances was explained the term of free assent and clarified and if there were an agreement, we scheduled an attendance that was carried through in the office of the pharmacy. The analysis was based on the "phase of the medicine bag" from Dadér method of farmacoterapêutico. Generally, the community presented certain nimbleness in the knowledge about their box of domiciliary medicines and interest about the differentiated service proposed.
\end{abstract}

Key-words: domiciliary medicine; pharmacist; pharmaceutical care.

\section{INTRODUÇÃO}

O conceito de Atenção Farmacêutica é "um modelo de prática farmacêutica, desenvolvida no contexto da Assistência Farmacêutica. Compreende atitudes, valores éticos, comportamento, habilidades, compromissos coresponsabilidades na prevenção de doenças, promoção e recuperação da saúde, de forma integrada a equipe de saúde" (IVAMA et al., 2002).

A automedicação é uma prática bastante difundida não apenas no Brasil. Contudo, mesmo na maioria dos países industrializados, vários medicamentos de uso mais simples e comum estão disponíveis em farmácias, drogarias ou supermercados, e podem ser obtidos sem necessidade de receita médica (CARAMELLI et al, 2001). O fato do indivíduo saber a forma correta de manusear os medicamentos é de suma importância, pois reduz o risco do uso de medicamento vencido, administrar doses ou posologia incorretas, armazenar em local 
indevido, recomendação terapêutica errônea dentre outros. Atualmente se observa uma carência de informações disponíveis sobre os medicamentos na comunidade, portanto é "obrigação do farmacêutico", o qual tem contato direto com o usuário, atender e esclarecer as dúvidas e anseios dos mesmos. Isso deve motivar as pessoas a levarem sua "farmácia domiciliar" até a farmácia comercial, que deve ser um ponto de referência em saúde e informações, e onde se encontra o farmacêutico, que exemplificará os cuidados que o indivíduo precisa ter com os medicamentos.

O intuito do estudo foi conhecer alguns aspectos técnicos em relação aos medicamentos que os usuários dos serviços de uma farmácia do município de Cascavel - Pr guardam em suas residências, o modo como vêm utilizando os mesmos, também, fornecer à comunidade orientação e informação sobre cada medicamento que possuem em casa, desfazendo dúvidas que poderiam ocasionar outros problemas de saúde, e indiretamente, demonstrar aos clientes de farmácia "comercial" o quão importante é utilizar-se dos conhecimentos do farmacêutico que realiza a orientação farmacêutica como parte da atenção farmacêutica, um serviço diferenciado e ainda pouco conhecido.

\section{MATERIAL E MÉTODOS}

Para a realização da pesquisa foi feito um convite aos clientes de uma farmácia comercial do município de Cascavel - Pr, esclarecendo-os sobre o objetivo do estudo, que seria a análise farmacêutica dos medicamentos da caixinha que eles têm em suas residências, no intuito de podermos fornecer informações e tirar dúvidas existentes. O convite foi realizado a todos os clientes que buscavam atendimento no estabelecimento até completar 15 aceites. O atendimento foi agendado e realizado no escritório da farmácia, no período de setembro a novembro de 2006.

A avaliação dos medicamentos da caixinha foi baseada na "fase da sacola de medicamentos" do método Dadér de acompanhamento farmacoterapêutico (MACHUCA, FERNÁNDEZ-LLIMÓS, FAUS, 2003), também foi verificada a validade do medicamento e aparência (sujidades, deformações e outros). Conforme fossem detectados erros ou dúvidas, seriam dirimidos baseados em literatura científica e conhecimento técnico e repassadas com linguagem acessível ao paciente, assegurando por meio de solicitação de repetição da informação, que estas foram entendidas.

\section{RESULTADOS E DISCUSSÃO}

Os clientes que aceitaram participar do estudo o fizeram, em geral, com muito interesse, existiram alguns questionamentos em relação a cobrança do serviço, após esclarecimentos de que não seria cobrado nada, houve adesão. Alguns clientes diziam não precisar de tais informações, porém retornavam a farmácia com prescrição médica e questionavam o serviço diferenciado, sendo realizado o estudo a partir deste interesse. Houve uma entrevista realizada na residência do paciente, por solicitação da mesma, exatamente em um dia que a mesma estava descartando seus medicamentos vencidos. A seguir destacamos os dados obtidos com o estudo.

- Sócio Demográfico: Foram entrevistados 13 mulheres (86\%) e dois homens (14\%), com média de idade de 40 anos (amplitude de 19 a 85 anos). Em relação à escolaridade cinco (34\%) têm o primário e cinco (34\%) têm o segundo grau completo, dos que têm nível superior completo são três (12\%) e dois (20\%) incompletos.

\section{ESCOLARIDADE}

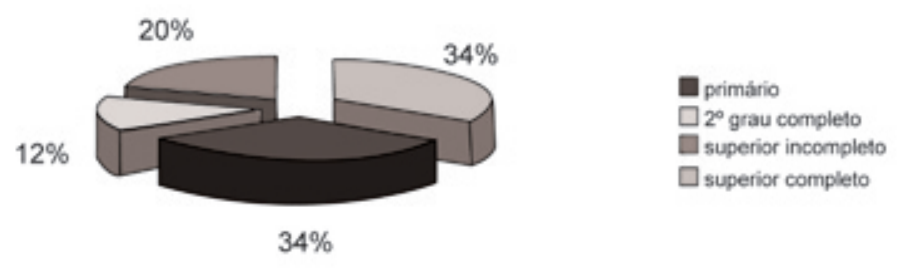

Figura 1 -Grau de escolaridade dos clientes atendidos

- Medicamentos: O total dos medicamentos avaliados foi 150, com média de dez por paciente. Constatou-se que um cliente com 19 medicamentos distintos e um caso com apenas um. Os medicamentos estudados foram organizados e classificados de acordo com o sistema ATC (Anatomical Therapeutic Chemical) (BARROSO, MORCILLO, TORRECILLA, 2005), em grupos representando o total geral e os subgrupos, representando a distribuição dentro do grupo, conforme disposto na tabela 1. 
Tabela 1 - Medicamentos utilizados no estudo classificados em porcentagem e valor absoluto conforme o sistema ATC

\begin{tabular}{|c|c|c|}
\hline GRUPO & $\%$ & $\mathrm{n}$ \\
\hline A: Aparelho digestório e metabolismo & 14,00 & 21 \\
\hline A02 Antiácidos, medicamentos para tratamento da úlcera péptica e da flatulência. & 14,00 & 03 \\
\hline A03 Agentes antiespasmódicos, anticolinérgicos e propulsores & 33,33 & 07 \\
\hline$\underline{\text { A04 Antieméticos e antinauseantes }}$ & 14,00 & 03 \\
\hline A06 Laxativos & 14,00 & 03 \\
\hline 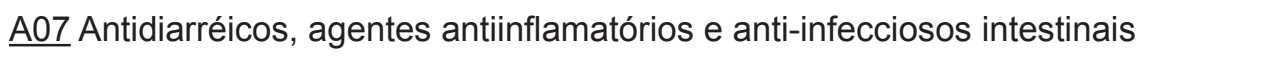 & 14,00 & 03 \\
\hline A11 Vitaminas & 4,76 & 01 \\
\hline$\underline{\mathrm{A} 12}$ Suplementos minerais & 4,76 & 01 \\
\hline B: Sangue e órgãos hematopoéticos & 3,33 & 05 \\
\hline B01 Medicamentos antitrombóticos & 80,00 & 04 \\
\hline B03 Preparados antianêmicos & 20,00 & 01 \\
\hline C: Aparelho cardiovascular & 7,30 & 11 \\
\hline C01 Terapêutica cardíaca & 9,09 & 01 \\
\hline$\underline{\text { C02 Anti-hipertensores }}$ & 27,27 & 03 \\
\hline$\underline{\text { C03 Diuréticos }}$ & 9,09 & 01 \\
\hline$\underline{\text { C07 Betabloqueadores }}$ & 9,09 & 01 \\
\hline C09 Agentes que atuam sobre o sistema renina-angiotensina & 18,18 & 02 \\
\hline$\underline{\text { C10 Hipolipemiantes }}$ & 27,27 & 03 \\
\hline GRUPO & $\%$ & $\mathrm{n}$ \\
\hline D: Medicamentos dermatológicos & 12,00 & 18 \\
\hline D01 Antifúngicos para uso dermatológico & 27,77 & 05 \\
\hline D02 Emolientes e protetores & 22,22 & 04 \\
\hline D06 Antibióticos e quimioterápicos para uso dermatológico & 16,66 & 03 \\
\hline D07 Corticosteroides, preparados dermatológicos_ & 27,77 & 05 \\
\hline D08 Anti-sépticos e desinfectantes & 5,55 & 01 \\
\hline G: Aparelho genito-urinário e hormônios sexuais & 5,33 & 08 \\
\hline G01Anti-infecciosos e anti-sépticos ginecológicos & 25,00 & 02 \\
\hline G03 Hormônios sexuais e moduladores do sistema genital & 75,00 & 06 \\
\hline H: Preparados hormonais sistêmicos, excluindo hormônios sexuais & 1,33 & 02 \\
\hline$\underline{\mathrm{H} 02}$ Corticosteróide para uso sistêmico & 100,00 & 02 \\
\hline J: Anti-infecciosos gerais para uso sistêmico_ & 8,66 & 13 \\
\hline J01 Antibacterianos para uso sistêmico_ & 76,92 & 10 \\
\hline J02 Antimicóticos para uso sistêmico_ & 15,38 & 02 \\
\hline$\underline{\text { J05 Antivirais para uso sistêmico_ }}$ & 7,69 & 01 \\
\hline
\end{tabular}




\begin{tabular}{|c|c|c|}
\hline$\underline{\text { M:Sistema músculo-esqueletico_ }}$ & 14,66 & 22 \\
\hline M01Anti-inflamatorio e anti-reumático & 59,09 & 13 \\
\hline M02 Produtos tópicos para dores articulares e musculares & 13,64 & 03 \\
\hline M03 Relaxantes musculares & 18,18 & 04 \\
\hline M05 Medicamentos para tratamento de doenças ósseas_ & 9,09 & 02 \\
\hline$\underline{\mathrm{N}}$ : Sistema nervoso & 21,33 & 32 \\
\hline N02 Analgésicos & 75,00 & 24 \\
\hline N04 Antiparkinsônicos & 3,12 & 01 \\
\hline$\underline{\text { N05 Psicolépticos }}$ & 18,75 & 06 \\
\hline N07 Outros medicamentos do sistema nervoso & 3,12 & 01 \\
\hline 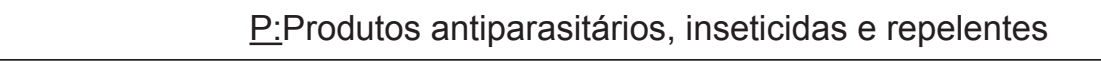 & 2,00 & 03 \\
\hline P01 Antiprotazoários & 66,66 & 02 \\
\hline P03 Ectoparasiticidas, incluindo escabicidas ${ }_{\perp}$ insecticidas e repelentes & 33,33 & 01 \\
\hline R:Aparelho respiratório_ & 7,33 & 11 \\
\hline$\underline{\mathrm{R} 01}$ Preparados para uso nasal & 36,36 & 04 \\
\hline$\underline{\text { R05 }}$ Preparados contra tosse e resfriados & 45,45 & 05 \\
\hline$\underline{\text { R06 Anti-histaminicos para uso sistêmico }}$ & 18,18 & 02 \\
\hline GRUPO & $\%$ & $\mathrm{n}$ \\
\hline S: Órgãos sensitivos & 2,66 & 04 \\
\hline S01Produtos oftalmológicos & 100 & 04 \\
\hline
\end{tabular}

Analisando os resultados podemos constatar que a maior porcentagem foi do grupo N (Sistema Nervoso), devido à expressiva participação do subgrupo dos analgésicos (N02), dados coincidentes com o estudo de Caramelli et al, (2001).

Todos os medicamentos apresentavam-se em bom estado de conservação, constatou-se que os pacientes têm consciência de onde guardá-los com segurança, fora do acesso a umidade, luz e calor. Os locais mais citados foram caixa de sapato, nécessaire, vasilhame, estes dentro do criado mudo ou do guarda-roupa.

Um aspecto importante quanto à estocagem dos medicamentos é o fato dos fármacos, para exercerem o máximo da ação benéfica desejada e o mínimo de efeitos adversos, têm necessidade de que se mantenham preservadas as condições de estabilidade, que é a propriedade de um produto em preservar - dentro de limites estabelecidos e sob determinadas condições ambientais - as mesmas características físicas, químicas e farmacológicas, durante seu período de vida útil. Esse espaço de tempo, no qual se assegura sua integridade, representa o período de validade (MARIN, 2003).

Sobre o uso contínuo, do total de 150 medicamentos, apenas $26(17,33 \%)$ eram regularmente administrados por $10(66,6 \%)$ pacientes entrevistados, enquanto os outros cinco $(33,4 \%)$ entrevistados não faziam uso de nenhum medicamento no momento (figura 2), considerando o fato de ter sido questionado se estava havendo utilização de algum deles por outro membro da família. Sendo assim, para os $124(82,6 \%)$ medicamentos restantes não havia utilização de forma contínua. 
USO DE MEDICAMENTOS

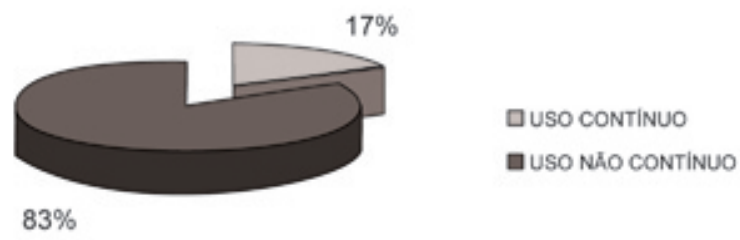

Figura 2 - Uso de medicamentos contínuo e não contínuo.

Desse total (124) houveram cinco justificativas para o não uso, sendo que $50(40,32 \%)$ medicamentos eram para prevenção, $30(24,19 \%)$ estavam vencidos, oito $(6,45 \%)$ abandonaram o tratamento por conta própria, $30(24,19 \%)$ alcançaram a melhora da patologia e três $(2,41 \%)$ possuíam a caixa para uma possível referência (figura 3).

\section{MOTIVO DO USO NÃO CONTINNUO DE MEDICAMENTOS}
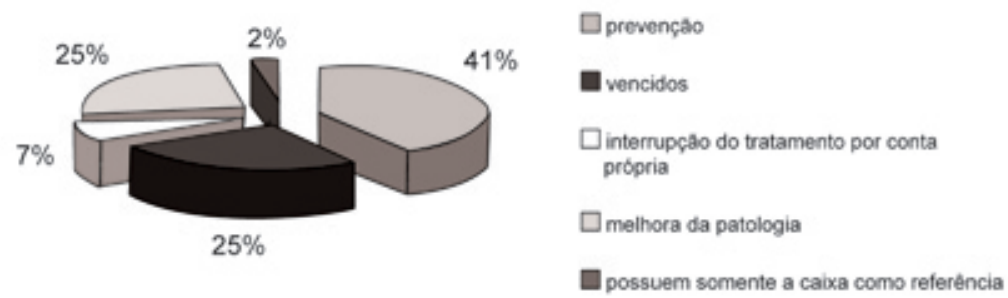

Figura 3 - Motivo do uso não continuo de medicamentos

De acordo com a Organização Mundial de Saúde, reação adversa a medicamento (RAM) é qualquer evento nocivo e não intencional que ocorreu na vigência do uso de um medicamento, utilizado com finalidade terapêutica, profilática ou diagnóstica, em doses normalmente recomendadas. Para minimizar os riscos de RAM e os custos com a utilização de medicamentos, estes devem ser usados de maneira racional (PFAFFENBACH et al, 2002).

Desse modo, podemos considerar como um risco a constatação desse significativo número de medicamentos estocados no domicílio de pacientes, que podem vir a ser utilizados incorretamente, gerando problemas de saúde.

Em relação à indicação, do total de 150, 130 (86,66\%) estavam sendo corretamente administrados, e 20 $(13,33 \%)$ erroneamente.

Em relação a responsabilidade pela indicação ou prescrição, 74 (50\%) do total foram automedicação realizada pelo próprio paciente, balconistas de farmácia, parentes ou vizinhos, 71 (47\%) por prescrição médica e cinco (3\%) por automedicação racional por meio do farmacêutico, com medicamentos de venda sem prescrição médica (figura 4).

Os entrevistados questionados sobre a posologia de seus medicamentos expressaram em 118 (78,66\%) dos casos a forma correta de administrá-los. O fato da maioria dos usuários saberem prontamente a posologia é importante, mas não garante que haja adesão ao tratamento. 


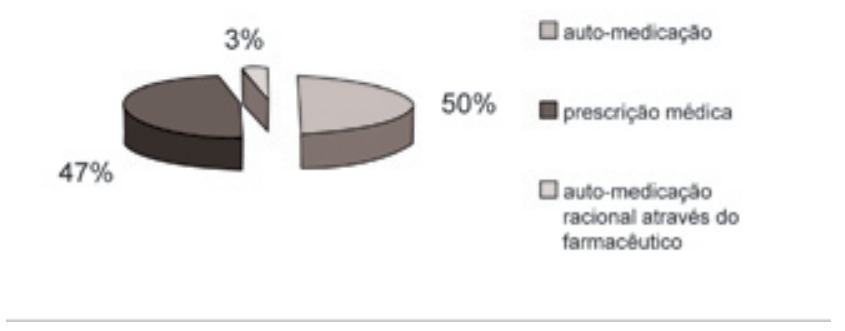

Figura 4 - Indicação ou prescrição do medicamento

Observou-se nesse estudo que, o nível de escolaridade não significa que o indivíduo possua entendimento em relação aos medicamentos presentes em casa. Pois, o grupo de pacientes semi-analfabetos (cinco) teve mais desenvoltura em relação aos questionamentos, também foram os mais prestativos e interessados pelo serviço diferenciado, mesmo tendo todas as informações necessárias para o bom uso de seus medicamentos. Relataram que administram apenas o que for receitado pelo médico. No entanto, alguns estudos afirmam o contrário: "a responsabilidade pela não-adesão ao tratamento é definida como ignorância dos pacientes ou responsáveis por eles sobre a importância do tratamento, a pouca educação da população (presumindo que seria um comportamento típico de classes menos privilegiadas), ou como simples desobediência de "ordens médicas" (LEITE, VASCONCELLOS, 2003). Efetivamente, a adesão terapêutica pode ser perspectivada como um comportamento relacionado com a saúde/doença, de tal forma que a compreensão dos fatores que levam as pessoas a aderir ou não às recomendações médicas enquadra-se no âmbito da psicologia, sendo a não adesão o conceito tradicionalmente empregue para designar a falha em seguir as prescrições médicas (KLEIN; GONÇALVES, 2005).

Em todos os casos houve a orientação para o cuidado com medicamentos vencidos. Encontramos um medicamento para tratamento de distúrbio gastrointestinal (pantoprazol) com apenas um comprimido utilizado e data de validade de 2003. O paciente atualmente queixa-se de muita dor quando ingere certos tipos de alimentos e foi orientado a retomar o tratamento com nova consulta.

A data de validade mais antiga foi de 1998, o medicamento estava sendo guardado para paciente não esquecer o nome e foi orientada a fazer uma relação destes medicamentos em papel, pois por descuido poderia utilizá-los vencidos. Outra situação de erro foi um caso de dois antigripais em que não foi encontrada a data de vencimento, pois a usuária picota a cartela, a mesma foi orientada sobre os riscos, neste caso não foi autorizado o descarte do medicamento.

Outra situação foi o uso de um moderador de apetite, sibutramina, por prescrição médica, sendo que a usuária sentia-se bem, no entanto, confidenciou que havia desmaiado por falta de alimentação. Disse ter medo de contar o fato para o seu médico e o mesmo retirar a prescrição. A usuária foi orientada de que seria apropriado e seguro relatar o fato, para que fosse encontrada a solução correta.

Houve um caso restrito, porém preocupante, de uma caixa apresentar 49 comprimidos de amoxicilina e 10 de cefalexina, sendo todos amostras grátis recebidas em consultas médicas, a usuária relatou que o estoque serviria como prevenção de possíveis infecções. Na antibioterapia, o não cumprimento correto do tratamento resulta em um potencial risco a saúde pública, ao aumentar notavelmente a resistência de microorganismos aos antibióticos; a duração do tratamento tem um papel decisivo na erradicação dos microorganismos que produzem a infecção (MACHUCA et al, 2003). O desenvolvimento de resistência bacteriana, portanto, pode ser adquirida por meio da automedicação, distribuição de antimicrobianos por balconistas de farmácia ou até mesmo médica, ressaltando que quanto maior a dose e a duração do uso de antibióticos, maior o risco de colonização e infecção por bactérias multi-resistentes.

Destacamos alguns entrevistados, no total quatro dos clientes $(26,66 \%)$, que não utilizam medicamentos por qualquer coisa, pois dizem preferir "passar dor a tomar remédio". Compram apenas quando é prescrição médica. 


\section{CONSIDERAÇÕES FINAIS}

A abordagem serviu para mostrar que o papel do farmacêutico vai além da dispensação sem informação. Os colegas poderiam se surpreender ao saberem quantos desconhecem a importância e o alcance da profissão. É muito positivo saber que se promoveu o uso correto dos medicamentos por meio dos esclarecimentos prestados, e obter a satisfação de ser útil orientando para uma terapia farmacoterapêutica eficiente. Houveram alguns clientes que afirmaram sentir-se mais a vontade com o farmacêutico, demonstrando às vezes medo de contar alguma irregularidade que cometeram em seus tratamentos ou fatos da vida pessoal que poderiam alterar o mesmo. Apesar de certas dificuldades apresentadas, é válida a introdução desta prática no estabelecimento comercial, pois é nítida e poderia se dizer, urgente, a necessidade de informações técnicas de qualidade visando o uso racional de medicamentos, havendo melhora tanto para a farmácia como estabelecimento de saúde, quanto para o farmacêutico que obterá crescimento na vida profissional. Estará também compartilhando conhecimentos adquiridos na graduação, com pessoas que não tiveram oportunidade ou falta de tempo para poder entender a ciência dos medicamentos, melhorando a qualidade de vida dos pacientes.

\section{REFERÊNCIAS BIBLIOGRÁFICAS}

BARROSO, A.V.; MORCILLO, R.M.S.; TORRECILLA, E.M.I. Revision de Botiquines Domésticos de personas incluídas em Ayuda a Domiciliaria. 7 (1): 32-40. Pharmaceutical Care Espana: 2005.

CARAMELLI, B.; TEIXEIRA, C.; KASEER, C.; VILHENA, V. Editorial da Revista da Associação Médica Brasileira. 47 (4). São Paulo: ABM, 2001.

IVAMA, A.M.; NOBLAT, L.; CASTRO, M.S.; JARAMILLO, N.M.; OLIVEIRA, N.V.B.V; RECH, N. Consenso Brasileiro sobre Atenção Farmacêutica. Proposta. Brasília: Organização Pan-Americana de Saúde, 2002.

LEITE, S.N.; VASCONCELLOS, M.P.C. Adesão à terapêutica medicamentosa: elementos para a discussão de conceitos e pressupostos adotados na literatura. 8 (3): 775-782. Ciência \& Saúde Coletiva: 2003

MACHUCA, M.; ESPEJO, J.; GUTIERREZ, L.; MACHUCA, M.P.; HERRERA, J. La informacion escrita del farmaceutico mejora el cumplimento de la antibioterapia. 44 (2): 141 - 157. Ars Pharmaceutica: 2003.

MACHUCA, M., FERNANDEZ - LLIMOS, F., FAUS, M.J. Método Dader. Guia de seguimento farmacoterapeutico. GIAF - UGR, 2003.

MARIN, N.; LUIZA, V.L.; CASTRO, C.G.S.O.; SANTOS, S.M. Assistência farmacêutica para gerentes municipais. Rio de Janeiro: OPAS/OMS, 2003.

PFAFFENBACH, G.; CARVALHO, O. M.; BERGSTEN-MENDES, G. Reações Adversas a Medicamentos com Determinantes da Admissão Hospitalar. 48(3): 237-41. Rev Assoc Med Bras: 2002. 